\title{
Dynamic Parameter Identification of a 6 DOF Industrial Robot using Power Model
}

\author{
Maxime Gautier and Sébastien Briot
}

\begin{abstract}
Off-line dynamic identification requires the use of a model linear in relation to the robot dynamic parameters and the use of linear least squares technique to calculate the parameters. Most of time, the used model is the Inverse Dynamic Identification Model (IDIM). However, the computation of its symbolic expressions is extremely tedious. In order to simplify the procedure, the use of the Power Identification Model (PIM), which is dramatically simpler to obtain and that contains exactly the same dynamic parameters as the IDIM, was previously proposed. However, even if the identification of the PIM parameters for a 2 degrees-offreedom (DOF) planar serial robot was successful, its fails to work for 6 DOF industrial robots. This paper discloses the reasons of this failure and presents a methodology for the identification of the robot dynamic parameters using the PIM. The method is experimentally validated on an industrial 6 DOF Stäubli TX-40 robot.
\end{abstract}

\section{INTRODUCTION}

$\mathrm{S}$ EVERAL schemes have been proposed in the literature to identify the dynamic parameters of robots [1]-[7]. Most of the dynamic identification methods have the following common features:

- the use of a model linear in relation to the dynamic parameters,

- the construction of an over-determined linear system of equations obtained by sampling the model while the robot is tracking some trajectories in closed-loop control,

- the estimation of the parameter values using least squares techniques $(L S)$.

The experimental works have been carried out either on prototypes in laboratories or on industrial robots and have shown the benefits in terms of accuracy in many cases.

To carry out the identification of the dynamic parameters, the Inverse Dynamic Identification Model (IDIM) is usually used. However, the computation of its symbolic expressions is extremely tedious. In order to simplify the procedure, the use of the Power Identification Model (PIM), which is dramatically simpler to obtain and that contains exactly the same dynamic parameters as the IDIM, was previously proposed in [8]. The PIM was used by one of the authors of

Manuscript received September 17, 2012. This work has been partially funded by the French ANR project ARROW (ANR 2011 BS3 006 01).

M. Gautier is with the IRCCyN and with the LUNAM, University of Nantes, 44321 Nantes France (phone: +33(0)240376960; fax: +33(0)240376930; e-mail: Maxime.Gautier@irccyn.ec-nantes.fr).

S. Briot is with the French CNRS and the IRCCyN, 44321 Nantes France (e-mail: Sebastien.Briot@irccyn.ec-nantes.fr). the present paper for the identification of the dynamic parameters of 2 degrees-of-freedom (DOF) planar serial robot [8] but its application to a 6-DOF serial industrial robot was not successful and the results never published.

The reasons of this failure are disclosed in this paper. It will be shown that the PIM is much more sensitive to the choice of the exciting trajectories than the IDIM. In order to show the effectiveness of the PIM for the identification of inertial parameters of 6 DOF serial robots, the method is experimentally validated on an industrial Stäubli TX-40 robot and compared with the usual IDIM procedure.

The paper is organized as follows: sections 2 and 3 make some brief recalls on the computation of the IDIM and PIM. Section 4 discloses the identification procedure. Section 5 presents the experimental validations. Finally, section 6 gives the conclusion.

\section{The USUAL INVERSE DyNAmic MODELS}

The inverse dynamic model (IDM) of a rigid robot composed of $n$ moving links calculates the $(n \times 1)$ motor torque vector $\tau_{i d m}$, as a function of the generalized coordinates and their derivatives. It can be obtained from the Newton-Euler or the Lagrange equations [5], [9]. It is given by the following relation:

$\tau_{\text {idm }}=M(q) \ddot{q}+N(q, \dot{q})$

where $q, \dot{q}$ and $\ddot{q}$ are respectively the $(n \times 1)$ vectors of generalized joint positions, velocities and accelerations, $M(q)$ is the $(n \times n)$ robot inertia matrix, and $N(q, \dot{q})$ is the $(n \times 1)$ vector of centrifugal, Coriolis, gravitational and friction forces/torques.

It is known that the dynamic model of any manipulator with $n$ actuators can be linearly written in term of a $(n \times 1)$ vector of standard parameters $\chi_{s t}[1],[4],[5]$ :

$\tau_{i d m}\left(q, \dot{q}, \ddot{q}, \chi_{s t}\right)=I D M_{s t}(q, \dot{q}, \ddot{q}) \chi_{s t}$

where:

$I D M_{s t}$ is the $\left(n \times n_{s t}\right)$ jacobian matrix of $\tau_{i d m}$, with respect to the $\left(n_{s t} \times 1\right)$ vector $\chi_{s t}$ of the standard parameters given by $\chi_{s t}=\left[\begin{array}{llll}\chi_{s t}^{1 T} & \chi_{s t}^{2 T} & \ldots & \chi_{s t}^{n T}\end{array}\right]^{T}$.

For rigid robots, there are 14 standard parameters by link and joint. For the joint and link $j$, these parameters can be regrouped into the $(14 \times 1)$ vector $\chi_{s t}^{j}[5]$ : 
$\chi_{s}^{j}=\left[X X_{j} X Y_{j} X Z_{j} Y_{j} Z_{j} Z_{j} M X_{j} M M_{j} M_{j} M_{j} I a_{j} F v_{j} F c_{j} \tau_{d f j}\right]^{T}$

where:

$X X_{j}, X Y_{j}, X Z_{j}, Y Y_{j}, Y Z_{j}, Z Z_{j}$ are the 6 components of the inertia matrix of link $j$ at the origin of frame $j$.

$M X_{j}, M Y_{j}, M Z_{j}$ are the 3 components of the first moment of link $j, M_{j}$ is the mass of link $j, I a_{j}$ is a total inertia moment for rotor and gears of actuator $j$.

$F v_{j}, \quad F c_{j}$ are the visquous and Coulomb friction coefficients of the transmission chain, respectively,

$\tau_{\text {off }}=\tau_{\text {offF }}+\tau_{\text {off } \tau_{j}}$ is an offset parameter which regroups the amplifier offset $\tau_{o f f \tau_{j}}$ and the asymmetrical Coulomb friction coefficient $\tau_{\text {offFS }}$.

The identifiable parameters are the base parameters which are the minimum number of dynamic parameters from which the dynamic model can be calculated. They are obtained from the standard inertial parameters by regrouping some of them by means of linear relations [10], which can be determined for the serial robots using simple closed-form rules [3], [5], or by numerical method based on the QR decomposition [11].

The minimal dynamic model can be written using the $n_{b}$ base dynamic parameters $\chi$ as follows:

$\tau_{i d m}=\operatorname{IDM}(q, \dot{q}, \ddot{q}) \chi$

where $I D M$ is a subset of independent columns of $I D M_{s t}$ which defines the identifiable parameters. (4) takes the following block-triangular form:

$\left[\begin{array}{c}\tau_{1} \\ \tau_{2} \\ \vdots \\ \tau_{n-1} \\ \tau_{n}\end{array}\right]=\left[\begin{array}{ccccc}D^{1,1} & D^{1,2} & \cdots & D^{1, n-1} & D^{1, n} \\ 0 & D^{2,2} & \cdots & D^{2, n-1} & D^{2, n} \\ \vdots & \vdots & \vdots & \vdots & \vdots \\ 0 & 0 & \cdots & D^{n-1, n-1} & D^{n-1, n} \\ 0 & 0 & \cdots & 0 & D^{n, n}\end{array}\right]\left[\begin{array}{c}\chi^{1} \\ \chi^{2} \\ \vdots \\ \chi^{n-1} \\ \chi^{n}\end{array}\right]$

where $\tau_{i}$ is the input torque of actuator $i, \chi^{j}$ the base dynamic parameters of the joint $j$ and $D_{i j}$ the row vector of matrix IDM corresponding to the actuator $i$ and the parameters $\chi^{j}(i, j=1, \ldots, n)$.

Because of perturbations due to noise measurement and modelling errors, the actual force/torque $\tau$ differs from $\tau_{i d m}$ by an error, $e$, such that:

$\tau=\tau_{\text {idm }}+e=\operatorname{IDM}(q, \dot{q}, \ddot{q}) \chi+e$

where $\tau$ is calculated with the drive chain relations:

$\tau=v_{\tau} g_{\tau}=\left[\begin{array}{ccc}v_{\tau}^{1} & 0 & 0 \\ 0 & \ddots & 0 \\ 0 & 0 & v_{\tau}^{n}\end{array}\right]\left[\begin{array}{c}g_{\tau}^{1} \\ \vdots \\ g_{\tau}^{n}\end{array}\right]$

$v_{\tau}$ is the $(n \times n)$ matrix of the actual motor current references of the current amplifiers $\left(v_{\tau}^{j}\right.$ corresponds to actuator $j)$ and $g_{\tau}$ is the $(n \times 1)$ vector of the joint drive gains ( $g_{\tau}^{j}$ corresponds to actuator $j$ ) that is given by a priori manufacturer's data or identified [12][13]. Equation (6) represents the Inverse Dynamic Identification Model (IDIM).

\section{THE POWER MODEL}

In order to decrease the complexity of computing the symbolic expressions for the identification process, a model based on the energy has been proposed [8], [14] for the identification of a 2 DOF planar serial robot. This model can be obtained by calculating the power $P_{p m}$ of the system:

$P_{p m}=\frac{d}{d t}(H(q, \dot{q}))+\dot{q}^{T} \tau_{f}$

where $H(q, \dot{q})$ is the total energy of the system calculated using the recursive equations proposed in [5] and $\tau_{f}$ being the vector of the friction torques, i.e.

$\tau_{f}=\left[\tau_{f 1}, \tau_{f 2}, \ldots, \tau_{f n}\right]^{T}, \tau_{f j}=F v_{j} \dot{q}_{j}+F s_{j} \operatorname{sign}\left(\dot{q}_{j}\right)+\tau_{o f f j} .(9)$

The relation (8) can be expressed as a linear form with respect to the base dynamic parameters of the robot:

$P_{p m}=\frac{d}{d t}(h(q, \dot{q})) \chi=\frac{d}{d t}\left(\left[h^{1}, h^{2}, \ldots, h^{n}\right]\right)\left[\begin{array}{c}\chi^{1} \\ \chi^{2} \\ \vdots \\ \chi^{n}\end{array}\right]=d h \chi$

where $h(q, \dot{q})$ is the $\left(1 \times n_{b}\right)$ jacobian matrix of the energy with respect to the base dynamic parameters, $d h$ the $\left(1 \times n_{b}\right)$ jacobian matrix of the power with respect to the base dynamic parameters and $h^{j}$ the vector of matrix $h$ corresponding to the parameters $\chi^{j}(j=1, \ldots, n)$.

It must be mentioned here that, due the serial architecture of industrial robots, the vector $h_{j}$ depends on joint velocities $\dot{q}_{1}$ to $\dot{q}_{j}$ only and $h^{j}=0$ (as well as $h^{j}, d h^{j}$ being the vector of matrix $d h$ corresponding to $\chi^{j}$ ) if joints 1 to $j$ are fixed. This intrinsic property of matrices $h$ and $d h$ is crucial for the following of the paper.

Because of perturbations due to noise measurement and modelling errors, the robot power $P$ differs from $P_{p m}$ by an error, $e$, such that:

$P=P_{p m}+e=d h \chi+e$

where $P$ is calculated with:

$P=\dot{q}^{T} \tau$

(11) represents the Power Identification Model (PIM).

The $P I M$ is a scalar equation whose symbolic expressions are easier to derive than the vector expressions of the IDIM. 


\section{THE IDENTIFICATION PROCEDURE}

\section{A. Identification of the dynamic parameters}

The off-line identification of the base dynamic parameters $\chi$ is considered, given measured or estimated off-line data for $\tau$ or $P$ and $(q, \dot{q}, \ddot{q})$, collected while the robot is tracking some planned trajectories.

For the IDIM, $(q, \dot{q}, \ddot{q})$ in (6) are estimated with $(\hat{q}, \hat{\dot{q}}, \hat{q})$, respectively, obtained by band-pass filtering the measure of $q$ [8]. For the PIM, $(q, \dot{q})$ and matrix $d h$ in (10) are estimated with $(\hat{q}, \hat{\dot{q}})$ and $d \hat{h}$, respectively, obtained by band-pass filtering the measure of $q$ and values of $h(q, \dot{q})$. The principle is to sample the identification models (6) or (11) at a frequency $f_{m}$ in order to get an over-determined linear system of $r_{m}$ equations and $n_{b}$ unknowns such that:

$Y_{f m}=W_{f m} \chi+\rho_{f m}$

In order to cancel the high frequency torque ripple in $Y_{f m}$ and to window the identification frequency range into the model dynamics, a parallel decimation procedure low-pass filters in parallel $Y_{f m}$ and each column of $W_{f m}$ and resamples them at a lower rate, keeping one sample over $n_{d}$. This parallel filtering procedure can be carried out with the Matlab decimate function [8]. It is obtained:

$Y=W \chi+\rho$

$\rho$ is the $(r \times 1)$ vector of errors, with $r=r_{m} / n_{d}$,

$W$ is the $\left(r \times n_{b}\right)$ observation matrix.

Depending of what type of model is used, $Y$ is composed of the sampled data of either the measured torques $\tau$ (for the $I D I M$ ) or the estimated power $P=\dot{q}^{T} \tau$ (for the $P I M$ ). Similarly, $W$ concatenates either all matrices $I D M$ of (4) (for the $I D I M$ ) or all matrices $d h$ of (11) (for the PIM).

Using the base parameters and tracking "exciting" reference trajectories, a well-conditioned matrix $W$ is obtained. The $L S$ solution $\hat{\chi}$ of (14) is given by:

$\hat{\chi}=\left(\left(W^{T} W\right)^{-1} W^{T}\right) Y=W^{+} Y$

Standard deviations $\sigma_{\hat{\chi}_{i}}$, are estimated assuming that $W$ is a deterministic matrix and $\rho$, is a zero-mean additive independent Gaussian noise, with a covariance matrix $C_{\rho \rho}$, such that:

$C_{\rho \rho}=E\left(\rho \rho^{\mathrm{T}}\right)=\sigma_{\rho}^{2} I_{r}$

$E$ is the expectation operator and $I_{r}$, the $(r \times r)$ identity matrix. An unbiased estimation of the standard deviation $\sigma_{\rho}$ is:

$\hat{\sigma}_{\rho}^{2}=\|Y-W \hat{\chi}\|^{2} /(r-b)$

The covariance matrix of the estimation error is given by:
$C_{\hat{\chi} \hat{\chi}}=E\left[(\chi-\hat{\chi})(\chi-\hat{\chi})^{\mathrm{T}}\right]=\hat{\sigma}_{\rho}^{2}\left(W^{\mathrm{T}} W\right)^{-1}$.

$\sigma_{\hat{\chi}_{i}}^{2}=C_{\hat{\chi} \hat{\chi}}(i, i)$ is the $i^{\text {th }}$ diagonal coefficient of $C_{\hat{\chi} \hat{\chi}}$

The relative standard deviation $\% \sigma_{\hat{\chi}_{r i}}$ is given by:

$\% \sigma_{\hat{\chi}_{r i}}=100 \sigma_{\hat{\chi}_{i}} /\left|\hat{\chi}_{i}\right|$, for $\left|\hat{\chi}_{i}\right| \neq 0$

The ordinary $L S(O L S)$ can be improved by taking into account different standard deviations on equations errors [8]. In the case of the IDIM, each equation of joint $j$ in (14) is weighted with the inverse of the standard deviation of the error calculated from $O L S$ solution of the equations of joint $j$, in order to obtain the following system of equations that conserves the block-triangular form of (5):

$Y_{\tau}=\left[\begin{array}{c}Y_{\tau}^{l}\left(\tau^{1}\right) \\ Y_{\tau}^{2}\left(\tau^{2}\right) \\ \vdots \\ Y_{\tau}^{n-1}\left(\tau^{n-1}\right) \\ Y_{\tau}^{n}\left(\tau^{n}\right)\end{array}\right]=\left[\begin{array}{ccccc}W_{\tau}^{1,1} & W_{\tau}^{1,2} & \cdots & W_{\tau}^{1, n-1} & W_{\tau}^{1, n} \\ 0 & W_{\tau}^{2,2} & \cdots & W_{\tau}^{2, n-1} & W_{\tau}^{2, n} \\ \vdots & \vdots & \ddots & \vdots & \vdots \\ 0 & 0 & \cdots & W_{\tau}^{n-1, n-1} & W_{\tau}^{n-1, n} \\ 0 & 0 & \cdots & 0 & W_{\tau}^{n, n}\end{array}\right]\left[\begin{array}{c}\chi^{1} \\ \chi^{2} \\ \vdots \\ \chi^{n-1} \\ \chi^{n}\end{array}\right]+\rho_{\tau}$

where $Y_{\tau}^{j}\left(\tau^{j}\right)$ regroups the sampled and filtered values of the joint $j$ input torques and $W_{\tau}^{j, k}$ regroups the sampled and filtered values of vectors $D_{j, k}$ of (5).

For the PIM, the observation matrix has no blocktriangular form:

$Y_{P}\left(P_{p m}\right)=W_{P} \chi+\rho_{P}=\left[\begin{array}{lllll}W_{P}^{1} & W_{P}^{2} & \cdots & W_{P}^{n-1} & W_{P}^{n}\end{array}\right]\left[\begin{array}{c}\chi^{1} \\ \chi^{2} \\ \vdots \\ \chi^{n-1} \\ \chi^{n}\end{array}\right]+\rho_{P}$

where $Y_{P}\left(P_{p m}\right)$ regroups the sampled and filtered values of the power $P_{p m}$ and $W_{P}^{k}$ regroups the sampled and filtered values of vectors $d h^{k}$ of (10).

Furthermore, for both the IDIM and PIM, if the data collected on different trajectories are concatenated in (14), the equations corresponding to one given trajectory can be weighted using the same procedure, and that for all the concerned trajectories.

This weighting operation normalises the errors in (14) and gives the weighted LS (IDIM-WLS or PIM-WLS) estimation of the parameters.

\section{B. Discussion about the exciting reference trajectories}

Due to the intrinsic nature of serial industrial robots, the inertial parameters of the last joints (especially, those of the wrist) are the most difficult to identify. Indeed, the wrist elements are lighter and if their corresponding inertial parameters are melt in the same equation with those of the first joints, they will be poorly identified.

This problem is partially solved when using the IDIM procedure thanks to the block-triangular structure of the observation matrix shown in (20). The $L S$ solution of (20) minimizes the squared norm of the error $\rho_{\tau}$ : 

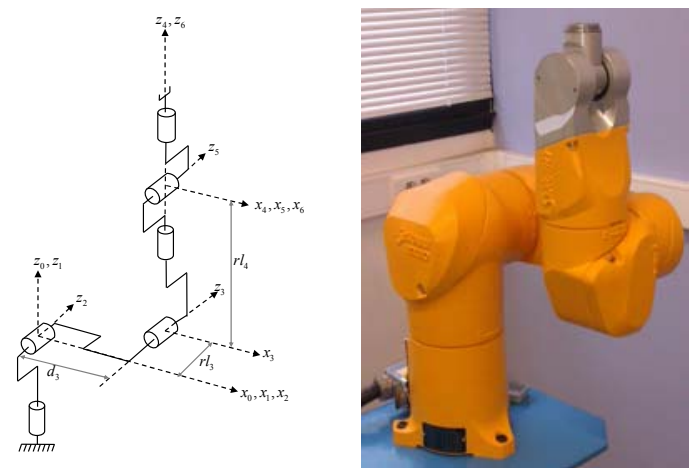

Fig. 1. Link frames of the TX-40 robot

TABLE I GEOMETRIC PARAMETERS OF THE TX-40 ROBOT WITH THE PAYLOAD

\begin{tabular}{lllllll}
\hline \hline$j$ & $\sigma_{j}$ & $\alpha_{j}$ & & $d_{j}$ & $\theta_{j}$ & $r_{j}$ \\
\hline 1 & 0 & 0 & 0 & $q_{1}$ & 0 \\
2 & 0 & $-\pi / 2$ & 0 & $q_{2}-\pi / 2$ & 0 \\
3 & 0 & 0 & $d_{3}=0.225 \mathrm{~m}$ & $q_{3}+\pi / 2$ & $r l_{3}=0.035 \mathrm{~m}$ \\
4 & 0 & $+\pi / 2$ & 0 & $q_{4}$ & $r l_{4}=0.225 \mathrm{~m}$ \\
5 & 0 & $-\pi / 2$ & 0 & $q_{5}$ & 0 \\
6 & 0 & $+\pi / 2$ & 0 & $q_{6}$ & 0 \\
7 & 2 & 0 & 0 & 0 & 0 \\
\hline \hline
\end{tabular}

$$
\begin{aligned}
\left\|\rho_{\tau}\right\|^{2} & =\left\|Y_{\tau}^{1}-W_{\tau}^{1,1} \chi^{1}-\ldots-W_{\tau}^{1, n} \chi^{n}\right\|^{2}+\ldots \\
& +\left\|Y_{\tau}^{n-1}-W_{\tau}^{n-1, n-1} \chi^{n-1}-W_{\tau}^{n-1, n} \chi^{n}\right\|^{2}+\left\|Y_{\tau}^{n}-W_{\tau}^{n, n} \chi^{n}\right\|^{2}
\end{aligned}
$$

where the term $\left\|Y_{\tau}^{j}-W_{\tau}^{j, j} \chi^{j}-\ldots-W_{\tau}^{j, n} \chi^{n}\right\|=\left\|\rho_{\tau}^{j}\right\|$ is the norm of the error on the estimation of the joint $j$ torque. Thus, minimizing the squared norm of $\rho_{\tau}$ is a stepwise coupled minimization of each squared norm of error $\rho_{\tau}^{j}$, starting from the parameters $\chi^{n}$. The squared norm $\left\|\rho_{\tau}^{n}\right\|^{2}$ in (22) contributes giving a good estimation of $\chi^{n}$, then the squared norm $\left\|\rho_{\tau}^{n-1}\right\|^{2}$ contributes giving a good estimation of the parameters $\chi^{n-1}$, etc.

Considering now the PIM, it can be directly observed that the observation matrix of (21) doesn't have a blocktriangular form and that the squared norm of the error $\rho_{P}$ is:

$\left\|\rho_{P}\right\|^{2}=\left\|Y_{P}-W_{P}^{l} \chi^{l}-\ldots-W_{P}^{n} \chi^{n}\right\|^{2}$

As the wrist links are lighter, the contribution of the wrist actuators to the total robot power is quite small with regards to the shoulder power, i.e. for a 6 DOF robot, $W_{P}^{4} \chi^{4}+W_{P}^{5} \chi^{5}+W_{P}^{6} \chi^{6}<<W_{P}^{1} \chi^{1}+W_{P}^{2} \chi^{2}+W_{P}^{3} \chi^{3}$.

Thus, the $L S$ solution of (21) may lead to a poor estimation of the wrist parameters.

Minimizing (23) using the PIM-WLS procedure should be compared with the IDIM-WLS procedure using only the joint 1 data in (22), i.e. the squared norm of the error for joint 1 , $\left\|\rho_{\tau}^{l}\right\|^{2}=\left\|Y_{\tau}^{1}-W_{\tau}^{1,1} \chi^{1}-\ldots-W_{\tau}^{1, n} \chi^{n}\right\|^{2}$.
This result will be shown in the next section. This problem can be avoided by creating a block-triangular regressor thanks to the use of optimal experimental trajectories. Using the property of matrices $d h$ mentioned in section III for a $n$-DOF industrial serial robot, the blocktriangular form of $W$ can be obtained by carrying out at least $n$ different types of trajectories that cancels some terms of $W$ :

1. Trajectories with all joints moving altogether

2. Trajectories with joint 1 fixed $\left(\dot{q}_{1}=0\right)$, all the other joints (from 2 to $n$ ) moving altogether

3. Trajectories with joints 1 and 2 fixed $\left(\dot{q}_{1}=\dot{q}_{2}=0\right)$, all the other joints (from 3 to $n$ ) moving altogether

$n$. Trajectories with joints 1 to $n-1$ fixed $\left(\dot{q}_{1}=\dot{q}_{2}=\ldots\right.$ $=\dot{q}_{n-1}=0$ ), joint $n$ moving only.

Using these trajectories, the observation matrix built with the PIM takes block-triangular form. In the next section, the PIM-WLS identification procedure is compared with the $I D I M-W L S$ procedure in order to show its efficiency.

\section{CASe Study}

\section{A. Description of the TX 40 kinematics}

The Stäubli TX-40 robot (Fig. 1) has a serial structure with six rotational joints. Its kinematics is defined using the modified Denavit and Hartenberg notation $(M D H)$ [15]. In this notation, the link $j$ fixed frame is defined such that the $z_{j}$ axis is taken along joint $j$ axis and the $x_{j}$ axis is along the common normal between $z_{j}$ and $z_{j+1}$ (Fig. 1). The geometric parameters defining the robot frames are given in Table 1. The payload is denoted as the link 7 . The parameter $\sigma_{j}=0$, means that joint $j$ is rotational, $\alpha_{j}$ and $d_{j}$ parameterize the angle and distance between $z_{j-1}$ and $z_{j}$ along $x_{j-1}$, respectively, whereas $\theta_{j}$ and $r_{j}$ parameterize the angle and distance between $x_{j-1}$ and $x_{j}$ along $z_{j}$, respectively. For link $7, \sigma_{j}=2$ means that the link 7 is fixed on the link 6 . Since all the joints are rotational then $\theta_{j}$ is the position variable $q_{j}$ of joint $j$.

The TX-40 robot is characterized by a coupling between the joints 5 and 6 such that:

$\left[\begin{array}{l}\dot{q} r_{5} \\ \dot{q} r_{6}\end{array}\right]=\left[\begin{array}{l}N_{5}=45 \\ N_{6}=32 N_{6}=32\end{array}\right]\left[\begin{array}{l}\dot{q}_{5} \\ \dot{q}_{6}\end{array}\right],\left[\begin{array}{l}\tau_{c_{5}} \\ \tau_{c_{6}}\end{array}\right]=\left[\begin{array}{cc}N_{5} & N_{6} \\ 0 & N_{6}\end{array}\right]\left[\begin{array}{l}\tau_{r_{5}} \\ \tau_{r_{6}}\end{array}\right]$

where $\dot{q} r_{j}$ is the velocity of the rotor of motor $j, \dot{q}_{j}$ is the velocity of joint $j, N_{j}$ is the transmission gain ratio of axis $j$, $\tau_{c j}$ is the motor torque of joint $j$, taking into account the coupling effect on the motor side, $\tau_{r j}$ is the electro-magnetic torque of motor $j$. 
TABLE II

IDENTIFIED DYNAMIC PARAMETERS.

\begin{tabular}{|c|c|c|c|c|c|c|c|c|c|c|c|c|}
\hline \multirow[b]{2}{*}{ Par. } & \multicolumn{2}{|c|}{ 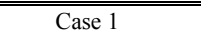 } & \multicolumn{2}{|c|}{ Case 2} & \multicolumn{4}{|c|}{ Case 3} & \multicolumn{4}{|c|}{ Case 4} \\
\hline & Values & $\% \sigma_{\hat{\chi}_{n}}$ & Values & $\% \sigma_{\hat{\chi}_{r}}$ & Values & $\% \sigma_{\hat{\chi}_{n}}$ & $\% e_{i 1}$ & $\% e_{i 2}$ & Values & $\% \sigma_{\hat{\chi}_{n}}$ & $\% e_{i}$ & $\% e_{i 2}$ \\
\hline$z z_{l r}$ & $1,29 \mathrm{e}+00$ & 0,45 & $1,27 \mathrm{e}+00$ & 0,36 & $1,18 \mathrm{e}+00$ & 2,40 & 8,53 & 7,09 & $1,24 \mathrm{e}+00$ & 1,84 & 3,88 & 2,36 \\
\hline$f v_{l}$ & $6,90 \mathrm{e}+00$ & 0,78 & $6,90 \mathrm{e}+00$ & 0,58 & $7,98 \mathrm{e}+00$ & 1,73 & 15,65 & 15,65 & $5,33 \mathrm{e}+00$ & 4,54 & 22,75 & 22,75 \\
\hline$f s_{1}$ & $6,71 \mathrm{e}+00$ & 2,37 & $6,72 \mathrm{e}+00$ & 1,76 & - & - & - & - & $1,59 \mathrm{e}+01$ & 6,45 & 136,96 & 136,61 \\
\hline$x x_{2 r}$ & $-4,57 \mathrm{e}-01$ & 2,02 & $-4,84 \mathrm{e}-01$ & 1,44 & $-4,68 \mathrm{e}-01$ & 6,26 & 2,41 & 3,31 & $-4,22 \mathrm{e}-01$ & 9,19 & 7,66 & 12,81 \\
\hline$x y_{2}$ & - & - & - & - & $-1,06 \mathrm{e}-01$ & 21,66 & - & - & - & - & - & - \\
\hline$x z_{2 r}$ & $-1,35 \mathrm{e}-01$ & 4,3 & $-1,45 \mathrm{e}-01$ & 3,03 & $-1,32 \mathrm{e}-01$ & 9,45 & 2,22 & 8,97 & $-1,22 \mathrm{e}-01$ & 12,79 & 9,63 & 15,86 \\
\hline$z z_{2 r}$ & $1,06 \mathrm{e}+00$ & 0,57 & $1,06 \mathrm{e}+00$ & 0,31 & $1,24 \mathrm{e}+00$ & 1,97 & 16,98 & 16,98 & $1,09 \mathrm{e}+00$ & 1,25 & 2,83 & 2,83 \\
\hline$m x_{2 r}$ & $2,22 \mathrm{e}+00$ & 0,52 & $2,21 \mathrm{e}+00$ & 0,27 & $2,02 \mathrm{e}+00$ & 1,95 & 9,01 & 8,60 & $2,18 \mathrm{e}+00$ & 0,98 & 1,8 & 1,36 \\
\hline$f v_{2}$ & $4,54 \mathrm{e}+00$ & 1,33 & $4,45 \mathrm{e}+00$ & 0,72 & $1,42 \mathrm{e}+00$ & 26,46 & 68,72 & 68,09 & $4,28 \mathrm{e}+00$ & 3,19 & 5,73 & 3,82 \\
\hline$f_{s_{2}}$ & $8,11 \mathrm{e}+00$ & 1,83 & $7,87 \mathrm{e}+00$ & 0,97 & $2,80 \mathrm{e}+01$ & 7,18 & 245,25 & 255,78 & $8,25 \mathrm{e}+00$ & 6,68 & 1,73 & 4,83 \\
\hline$x x_{3 r}$ & - & - & $9,49 \mathrm{e}-02$ & 7,62 & - & - & - & - & - & - & - & - \\
\hline$x z_{3}$ & - & - & - & - & 0,093 & 23,48 & - & - & - & - & - & - \\
\hline$y z_{3}$ & - & - & - & - & 0,225 & 10,06 & - & - & - & - & - & - \\
\hline$z z_{3 r}$ & $1,39 \mathrm{e}-01$ & 3,7 & $1,46 \mathrm{e}-01$ & 1,81 & $2,70 \mathrm{e}-01$ & 10,47 & 94,24 & 84,93 & $1,57 \mathrm{e}-01$ & 6,56 & 12,95 & 7,53 \\
\hline$m y_{3 r}$ & $-6,30 \mathrm{e}-01$ & 1,53 & $-6,07 e-01$ & 0,79 & - & - & - & - & $-6,10 e-01$ & 1,5 & 3,17 & 0,49 \\
\hline$i a_{3}$ & $8,27 \mathrm{e}-02$ & 5,83 & $8,74 \mathrm{e}-02$ & 2,85 & - & - & - & - & $7,54 \mathrm{e}-02$ & 12,88 & 8,83 & 13,73 \\
\hline$f v_{3}$ & $1,73 \mathrm{e}+00$ & 2,61 & $1,60 \mathrm{e}+00$ & 1,19 & - & - & - & - & $1,32 \mathrm{e}+00$ & 3,47 & 23,7 & 17,5 \\
\hline$f_{s_{3}}$ & $6,30 \mathrm{e}+00$ & 2,4 & $6,30 \mathrm{e}+00$ & 1,03 & $2,08 \mathrm{e}+01$ & 3,35 & 230,16 & 230,16 & $7,50 \mathrm{e}+00$ & 3,36 & 19,05 & 19,05 \\
\hline$x y_{4}$ & - & - & - & - & $-0,1$ & 11,09 & - & - & - & - & - & - \\
\hline$y z_{4}$ & - & - & - & - & 0,0588 & 19,79 & - & - & - & - & - & - \\
\hline$z z_{4 r}$ & - & - & - & - & - & - & - & - & $3,72 \mathrm{e}-02$ & 4,48 & - & - \\
\hline$m x_{4}$ & - & - & - & - & - & - & - & - & $-4,27 \mathrm{e}-02$ & 12,67 & - & - \\
\hline $\mathrm{ia}_{4}$ & - & - & $3,51 \mathrm{e}-02$ & 3,81 & - & - & - & - & - & - & - & - \\
\hline$f v_{4}$ & $9,15 \mathrm{e}-01$ & 4,79 & $8,51 \mathrm{e}-01$ & 1,78 & $8,56 \mathrm{e}-01$ & 9,90 & 6,45 & 0,59 & $7,34 \mathrm{e}-01$ & 3,02 & 19,78 & 13,75 \\
\hline$f_{s_{4}}$ & $2,40 \mathrm{e}+00$ & 6,87 & $2,55 \mathrm{e}+00$ & 2,31 & - & - & - & - & $3,05 \mathrm{e}+00$ & 4,56 & 27,08 & 19,61 \\
\hline$y z_{5}$ & - & - & - & - & 0,0561 & 17,15 & - & - & - & - & - & - \\
\hline$i a_{5}$ & $5,44 \mathrm{e}-02$ & 9,94 & $4,16 \mathrm{e}-02$ & 4,84 & $1,96 \mathrm{e}-01$ & 11,86 & 260,29 & 371,15 & $4,41 \mathrm{e}-02$ & 7,47 & 18,93 & 6,01 \\
\hline$f v_{5}$ & $1,60 \mathrm{e}+00$ & 3,8 & $1,56 \mathrm{e}+00$ & 1,42 & $3,02 \mathrm{e}+00$ & 4,09 & 88,75 & 93,59 & $1,46 \mathrm{e}+00$ & 3,08 & 8,75 & 6,41 \\
\hline$f_{s_{5}}$ & $3,37 \mathrm{e}+00$ & 4,47 & $2,71 \mathrm{e}+00$ & 1,92 & - & - & - & - & $2,00 \mathrm{e}+00$ & 9,54 & 40,65 & 26,2 \\
\hline$x y_{6}$ & - & - & - & - & 0,0182 & 21,67 & - & - & - & - & - & - \\
\hline$x z_{6}$ & - & - & - & - & - & - & - & - & $-2,54 \mathrm{e}-03$ & 22,05 & - & - \\
\hline$z z_{6}$ & - & - & - & - & $-0,026$ & 11,49 & - & - & - & - & - & - \\
\hline$m x_{6}$ & - & - & - & - & 0,055 & 17,75 & - & - & $-2,53 \mathrm{e}-02$ & 10,48 & - & - \\
\hline$m y_{6}$ & - & - & - & - & 0,0547 & 19,12 & - & - & - & - & - & - \\
\hline$i a_{6}$ & - & - & $1,09 \mathrm{e}-02$ & 4,43 & - & - & - & - & $9,99 \mathrm{e}-03$ & 2,76 & - & 8,35 \\
\hline$f v_{6}$ & $5,81 \mathrm{e}-01$ & 4,91 & $5,13 \mathrm{e}-01$ & 1,69 & $1,29 \mathrm{e}+00$ & 6,36 & 122,03 & 151,46 & $4,01 \mathrm{e}-01$ & 1,81 & 30,98 & 21,83 \\
\hline$f s_{6}$ & $1,96 \mathrm{e}+00$ & 7,72 & $1,83 \mathrm{e}+00$ & 2,68 & $-8,58 \mathrm{e}+00$ & 11,04 & 537,76 & 568,85 & $2,63 \mathrm{e}+00$ & 2,97 & 34,18 & 43,72 \\
\hline fvm $_{6}$ & $5,19 \mathrm{e}-01$ & 4,59 & $4,92 \mathrm{e}-01$ & 1,69 & $8,08 \mathrm{e}-01$ & 8,09 & 55,68 & 64,23 & $3,39 \mathrm{e}-01$ & 3,05 & 34,68 & 31,1 \\
\hline $\mathrm{fsm}_{6}$ & $1,85 \mathrm{e}+00$ & 7,76 & $1,53 \mathrm{e}+00$ & 3,19 & $-2,97 \mathrm{e}+00$ & 32,24 & 260,54 & 294,12 & $2,79 \mathrm{e}+00$ & 4,41 & 50,81 & 82,35 \\
\hline
\end{tabular}

$\sigma_{\hat{\chi}_{i}}$ is the standard deviation and $\% \sigma_{\dot{\chi}_{n}}$ its relative value (\%). $\% e_{i l}$ is the relative difference (\%) between the parameters identified in Case 1 and those identified with the PIM-WLS. $\% e_{i 2}$ is the relative difference (in \%) between the parameters identified in Case 2 and those identified with the PIM-WLS.

The coupling between joints 5 and 6 also adds the effect of the inertia of rotor 6 and new viscous and Coulomb friction parameters $\mathrm{Fvm}_{6}$ and $\mathrm{Fcm}_{6}$, to both $\tau_{c 5}$ and $\tau_{c 6}$.

It is possible to write:

$\tau_{c_{5}}=\tau_{5}+I a_{6} \ddot{q}_{6}+F v m_{6} \dot{q}_{6}+F c m_{6} \operatorname{sign}\left(\dot{q}_{6}\right)$ and

$\tau_{c_{6}}=\tau_{6}+I a_{6} \ddot{q}_{5}+F v m_{6} \dot{q}_{5}+F c m_{6}\left(\operatorname{sign}\left(\dot{q}_{5}+\dot{q}_{6}\right)-\operatorname{sign}\left(\dot{q}_{6}\right)\right)$ where $\tau_{j}$ already contains the terms $\left(I a_{j} \ddot{q}_{j}+F v_{j} \dot{q}_{j}+F c_{j} \operatorname{sign}\left(\dot{q}_{j}\right)\right)$, for $j=5$ and 6 respectively,

with $I a_{5}=N_{5}^{2} J a_{5}+N_{6}^{2} J a_{6}$ and $I a_{6}=N_{6}^{2} J a_{6}$

$J a_{j}$ is the moment of inertia of rotor $j$.

(26) is introduced into (4), (8) to obtain the IDIM and PIM.

\section{B. Identification results}

In this section, the identification procedure using PIM is compared with the usual method using IDIM. Three cases will be tested:

- Case 1: the robot dynamic parameters are identified with usual $I D I M-W L S$, using a single exciting trajectory with all joints moving simultaneously;

- Case 2: the robot dynamic parameters are identified with usual IDIM-WLS, using optimal trajectories presented in section IV.B;

- Case 3: the robot dynamic parameters are identified with PIM-WLS using the same trajectory as for Case 1;

- Case 4: the robot dynamic parameters are identified with PIM-WLS using the same trajectory as for Case 2.

Some small parameters remain poorly identifiable because they have no significant contribution in the joint torques. These parameters have no significant estimations and can be cancelled in order to simplify the dynamic model. Thus parameters such that the relative standard deviation $\% \sigma_{\hat{\chi}_{r i}}$ is too high are cancelled to keep a set of essential parameters of a simplified dynamic model with a good accuracy [16]. The essential parameters are calculated using an iterative

TABLE III

QUALITY OF IDENTIFICATION

\begin{tabular}{lrrrr}
\hline \hline & Case 1 & Case 2 & Case 3 & Case 4 \\
\hline Rel. Err. norm $\|\hat{\rho}\| /\|Y\|$ & 0.077358 & 0,0821229 & 0,0689218 & 0,0670227 \\
mean $\left(\% e_{i 1}\right)$ & - & - & 119.09 & 22.89 \\
mean $\left(\% e_{i 2}\right)$ & - & - & 131.97 & 21.70 \\
\hline \hline
\end{tabular}

$\|\hat{\rho}\|=\|Y-W \hat{\chi}\|$ is the minimal norm of error. 

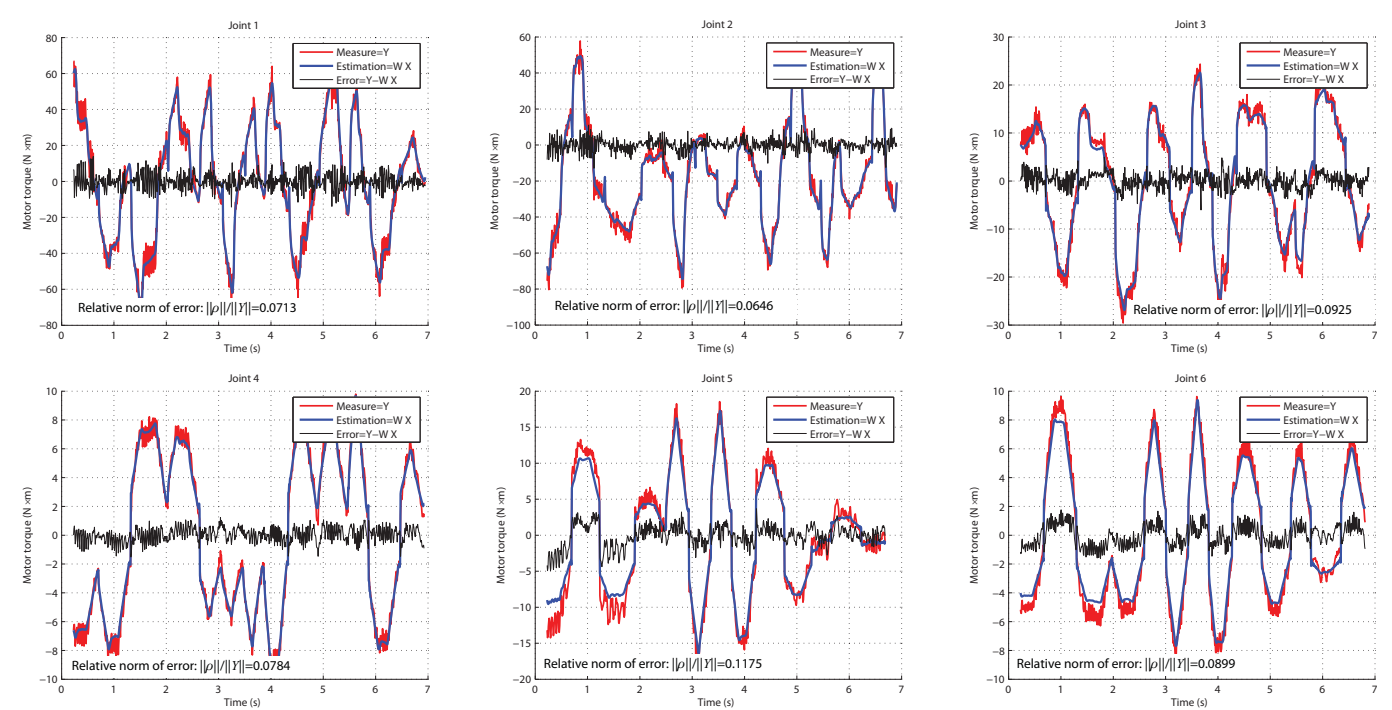

Fig. 2. Measured and reconstructed torques of the TX-40 with the parameters identified in Case 2.
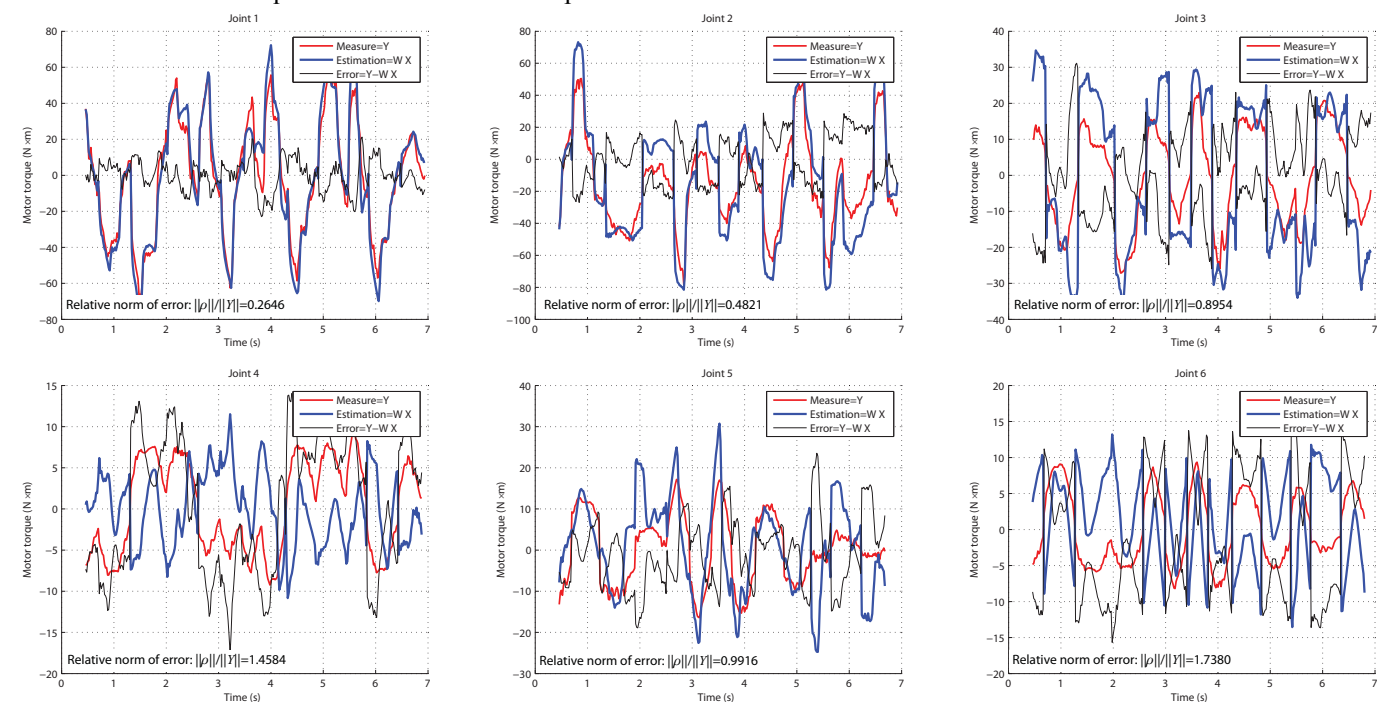

Fig. 3. Measured and reconstructed torques of the TX-40 with the parameters identified in Case 3.
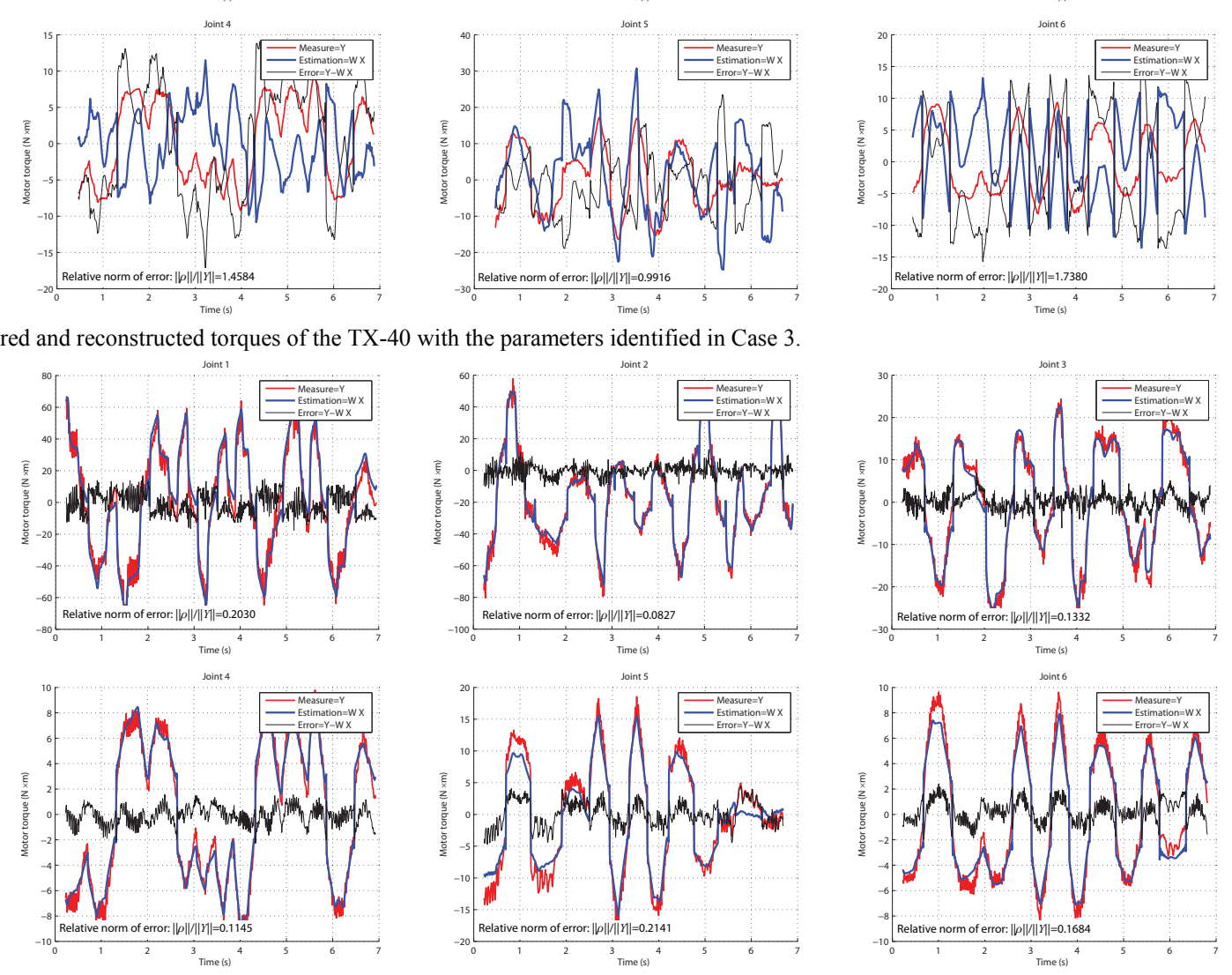

Fig. 4. Measured and reconstructed torques of the TX-40 with the parameters identified in Case 4. 
TABLE IV

QUALITY OF TORQUE RECONSTRUCTION. Case 1 Case 2 Case 3 Case 4 Case 5 \begin{tabular}{lllllll}
\hline Rel. Err. norm $\|\hat{\rho}\| /\|Y\|$ & 0.0726 & 0.0765 & 0.5346 & 0.1545 & 0.7485
\end{tabular}

procedure starting from the base parameters estimation. At each step the base parameter which has the largest relative standard deviation is cancelled. A new $L S$ parameter estimation of the simplified model is carried out with new relative error standard deviation $\% \sigma_{\hat{\chi}_{n}}$. The procedure ends when $\max \left(\% \sigma_{\hat{\chi}_{r i}}\right) / \min \left(\% \sigma_{\hat{\chi}_{r i}}\right)<r_{\sigma}$, where $r_{\sigma}$ is a ratio ideally chosen between 10 and 30 depending on the level of perturbation in $Y$ and $W$. Here, for all identification procedures, $r_{\sigma}$ is fixed to 20 .

The obtained results are shown in Table 2 . The parameters with the subscript $R$ stand for the regrouped parameters [3]. The results show that, in general, the parameters identified with PIM-WLS and optimized trajectories (Case 4) are closer to the parameters identified with IDIM-WLS (Cases 1 and 2). Some difference exists, but the parameters that have the largest differences $\% e_{i j}$ are those that have the largest relative standard deviation. It can also be observed that a larger number of parameters can be estimated when IDIM-WLS uses the trajectories optimized for PIM-WLS (Case 2), compared with the IDIM-WLS results obtained with a single trajectory (Case 1).

Table 3 presents the mean of the relative differences $\% e_{i j}$ between the parameter values estimated with PIM-WLS and $I D I M-W L S$. For the parameters estimated in Case 4, the mean of the difference with respect to those estimated in Cases 1 and 2 is stable and about $22 \%$. For the parameters estimated in Case 3, this value is from 6 times higher.

The relative error norm that gives an estimation of the quality of the identification procedure is also shown in the Table 3. Both PIM-WLS methods have a good identification quality, i.e. the identified parameters well estimate the robot power. However, only the IDIM-WLS and PIM-WLS procedure with optimized trajectories can correctly estimate the input torques (Fig. 2, 3 and 4; the reconstructed torques for Case 1 are not shown because the curves are very similar to those of Case 2), even if IDIM-WLS shows better results.

Finally, a last IDIM-WLS procedure is carried out to identify the robot parameters using the equation of joint 1 only (denoted as Case 5). The relative norm of error for each case of identification is shown in Table 4. The results show that, as mentioned in section IV.B., the torques are poorly reconstructed using both $P I M-W L S$ with a single trajectory and IDIM-WLS with the equations of joint 1 only, i.e. without the use of a block-triangular observation matrix.

Moreover, the quality of reconstruction is twice better with IDIM-WLS than with PIM-WLS. This can partially be explained by the fact that vector $Y_{P}$ in (21) is correlated with the observation matrix $W_{P}$ as they both depend of the estimated values of $\dot{q}$ in which there is noise. A possible solution to this problem is to adapt the procedure DIDIM [17] to the PIM, as this procedure uses simulated values (without noise) of $\dot{q}$. This is part of our future work.

\section{CONCLUSION}

This paper dealt with the identification of robot inertial parameters using the power model. This method uses a model with symbolic expressions dramatically simpler to compute than those of the usual inverse dynamic identification model, was formerly applied for the identification of the dynamic parameters of a planar 2-DOF serial robot but failed when applied to a 6-DOF serial industrial robot. The causes of this failure are disclosed in the present paper. It is shown that it is necessary to create a block-triangular observation matrix via the use of optimized trajectories in order to correctly identify the wrist inertial parameters. If not, the identification fails to find the parameters that are able to correctly estimate the actuator torques. The method has been experimentally validated on a Stäubli TX-40 robot and the results shown that this method is efficient for identifying the dynamic parameters of a 6 DOF industrial robot.

\section{REFERENCES}

[1] M. Gautier, "Identification of robots dynamics", Proc. IFAC Symp. on Theory of Robots, Vienne, Austria, December 1986, p. 351-356.

[2] C. Canudas de Wit and A. Aubin, "Parameters identification of robots manipulators via sequential hybrid estimation algorithms", Proc. IFAC Congress, Tallin, 1990, pp. 178-183.

[3] M. Gautier and W. Khalil, "Direct calculation of minimum set of inertial parameters of serial robots", IEEE TRO, Vol. 6, No. 3, 1990.

[4] J. Hollerbach, W. Khalil and M. Gautier, "Model Identification", chapter 14 « Springer Handbook of Robotics », Springer, 2008.

[5] W. Khalil and E. Dombre, "Modeling, identification and control of robots", Hermes Penton London, 2002.

[6] P.K. Khosla and T. Kanade, "Parameter identification of robot dynamics", Proc. $24^{\text {th }}$ IEEE CDC, 1985, p. 1754-1760.

[7] Z. Lu, K.B. Shimoga and A. Goldenberg, "Experimental determination of dynamic parameters of robotic arms", Journal of Robotics Systems, Vol. 10, Nº, 1993, p.1009-1029.

[8] M. Gautier, "Dynamic identification of robots with power model", Proc. IEEE Int. Conf. on Robotics and Automation, 1997, Albuquerque, New Mexico, April, pp. 1922-1927.

[9] R. Featherstone, D.E. Orin, "Dynamics", chapter 2 in B. Siciliano and O. Khatib. eds « Springer Handbook of Robotics », Springer, 2008.

[10] H. Mayeda, K. Yoshida and K. Osuka, "Base parameters of manipulator dynamic models", IEEE Trans. on Robotics and Automation, Vol. RA-6(3), 1990, p. 312-321.

[11] M. Gautier, "Numerical calculation of the base inertial parameters", Journal of Robotics Systems, Vol. 8, N4, 1991, pp. 485-506.

[12] P. Corke, "In situ measurement of robot motor electrical constants," Robotica, vol. 23, no. 14, pp.433-436, 1996.

[13] M. Gautier and S. Briot, "Global Identification of Drive Gains Parameters of Robots Using a Known Payload", Proceedings of the 2012 International Conference on Robotics and Automation (ICRA 2012), May 14-18, 2012, Saint Paul, MI, USA

[14] F. Reyes and R. Kelly, "Experimental Evaluation of Identifiation Schemes on a Direct Drive Robot," Robotica, 1997, Vol. 15, pp. 563571.

[15] W. Khalil and J.F. Kleinfinger, "A new geometric notation for open and closed loop robots", Proceedings of the IEEE International Conference on Robotics and Automation, 1986, San Francisco.

[16] C.M. Pham, M. Gautier, "Essential parameters of robots" Proceedings of the 30th Conference on Decision and Control, 1991, Brighton, England, December, pp. 2769-2774.

[17] M. Gautier, A. Janot and P.O. Vandanjon, "DIDIM: A New Method for the Dynamic Identification of Robots from only Torque Data," Proc. ICRA 2008, Pasadena, USA. 